\title{
Comunicación

\section{La amplificación social del riesgo: evidencias del accidente en la mina Pasta de Conchos}

\author{
NINA YOLANDA TEJEDA GARCÍA ${ }^{1}$ \\ LORENA PÉREZ-FLORIANO²
}

Dedicado a Javier Pérez, Amado Rosales, Jesús Morales, Lauro Olacio, Guillermo Iglesias, Adrián Barbosa, José Luis Calvillo, Oscar Javier Cerda, José Ángel Guzmán, Roberto Zapata y Mario Alberto Ruiz, Pedro Dueñez Posada, Ricardo Hernández Rocha, Jesús Rodríguez Torres, José Alberto de León, Fermín Narváez, José García, Rolando Alcocer, Roberto Guerrero, Gilberto Rico, Ignacio Hernández y Jorge Antonio Tovar; Juan Manuel Rosales, Jesús Álvarez, Agustín Botello, Jorge Bladimir Muñoz, Ignacio Campos, Juan Antonio Cruz y Juan Fernando García; Jesús Cortez Ibarra, Tomás Patlán, Juan Arturo Salazar, Felipe de Jesús Torres, Feliciano Vázquez, Pablo Soto, Hugo Ramírez, José Alfredo Ordóñez, Margarito Cruz Ríos y Gregorio Rangel; Margarito Zamarrón, Eliud Valero, Julián Martínez Ojeda, Raúl Villasana, Juan Antonio Cárdenas, Gilberto Ríos, Guillermo Ortiz, Mario Cordero, José Porfirio Sibrián, José Ramón Hernández, Juan Raúl Ortega, Luis Jorge de Hoyos y Arturo García; Mauro Antonio Sánchez, Ernesto de la Cruz, José Alfredo Silva, Jorge Arturo Ortega, Juan Ramón Barrientos, Juan Martín Gómez, Reyes Cuevas, José Armando Castillo, Isidoro Briceño, José Manuel Peña, José Isabel Mijares, Jesús Viera y José Eduardo Martínez. Mineros fallecidos en Pasta de Conchos.

1 Universidad Interamericana para el Desarrollo, del Consorcio Educativo Anahuac, México.

Correo electrónico:Tejeda.nina@gmail.com

2 Colegio de la Frontera Norte, México.

Correo electrónico: lorenapf@colef.mx 
La importancia que tiene para las organizaciones estar preparadas para una crisis y responder de forma expedita y competente fue puesta de manifiesto en el accidente industrial de la mina Pasta de Conchos, Coahuila, ocurrido en 2006. El accidente es analizado en este artículo a través de la teoría de amplificación de riesgos.

PALABRAS CLAVE: comunicación organizacional, minería, accidente industrial, comunicación en crisis, seguridad industrial, amplificación social de riesgo, mina Pasta de Conchos.
The importance of organizational crisis-readiness is made clear in the industrial accident of Pasta de Conchos mine, Coahuila, in 2006. The accident is analyzed through the Social Amplification Theory of Risk. Pasta de Conchos Management failed to respond on an expeditious and competent manner.

KEY WORDS: Organizational communication, mining, industrial accident, crisis communication, industrial safety, social amplifications of risks, Pasta de Conchos mine.

Durante el 2006, ocurrió un trágico accidente en la unidad de carbón Pasta de Conchos, en el Estado de Coahuila, México. La empresa dio respuesta inmediata y decisiva a este grave accidente, tanto en los trabajos de rescate y de búsqueda de nuestros mineros, como en el apoyo humanitario económico, educativo, de salud y social a las familias de nuestros mineros (Grupo México, 2006, p. 12).

\section{INTRODUCCIÓN}

El 19 de febrero del 2006 a las 2:20 a.m. se presentó un derrumbe en la mina Pasta de Conchos en el estado de Coahuila debido a la acumulación de gas grisú; derrumbe en el que 65 mineros perdieron la vida. Conforme pasaban los días, la situación de los mineros enterrados fue objeto de gran atención debido a los comunicados de la empresa, a distintas organizaciones gubernamentales y principalmente por los diversos medios de comunicación que cubrían el evento, el cual tuvo importantes implicaciones económicas, sociales, sindicales, legales y políticas.

En lo que se refiere al aspecto económico, la mina Pasta de Conchos dejó de producir 14,000 toneladas de carbón lavado por semana, lo que equivale a 1 millón 120 mil dólares. Las ventas anuales de la mina superaban los 50 millones de dólares. Cabe destacar que la falta de ventas no es la única perdida económica, Industria Minera México 
reportó el 17 de agosto que el costo de recuperación de los cuerpos de los 65 mineros ascendía a los 14 millones de dólares (Fernández, 2006). Además, posterior al derrumbe de la mina surgieron conflictos sindicales que provocaron un paro nacional en la industria minera y metalúrgica de México; las pérdidas derivadas ascendieron a más de 500 millones de dólares. Es necesario destacar que hubo reacciones sociales, principalmente en el municipio de Nueva Rosita, en donde los familiares, amigos y conocidos de los 65 mineros fallecidos exigieron tanto a las autoridades gubernamentales, como a los directivos de Grupo México una investigación e información acerca del rescate. Así mismo, hubo diferentes líderes involucrados, de la Iglesia, sindicales y políticos.

En este artículo se analiza el accidente industrial de la mina Pasta de Conchos a través de la teoría de amplificación social de riesgos (Kaspersone et al., 1988) que explica el fenómeno por el cual los procesos de información, las estructuras institucionales, el comportamiento de los grupos sociales y las respuestas individuales, dan forma a la experiencia social del riesgo y sus consecuencias.

Los objetivos de este artículo son: a) describir el accidente ocurrido en la mina de carbón Pasta de Conchos, b) aplicar la teoría de amplificación de riesgos para el análisis del accidente y c) hacer recomendaciones a partir del análisis realizado.

\section{LA TEORÍA DE AMPLIFICACIÓN DE RIESGOS}

Tres décadas de intensa investigación tanto teórica como metodológica rodean a la teoría de amplificación de riesgos. En los últimos años el rol del contexto y la cultura han sido investigados e incorporados a la teoría de amplificación de riesgos (Masuda \& Garvin, 2006; Rosa, 1998; Short, 1987; Wilkinson, 2001; Wynne, 1992). Cosmovisiones, redes sociales, orientación política y diferencias individuales son variables que se han estudiado para explicar quienes amplificarán y quienes atenuarán los riesgos. Masuda y Garvin (2006) demostraron que el espacio y las cosmovisiones culturales llevan a la amplificación del riesgo para las personas que viven en la comunidad en peligro y a la atenuación del riesgo para las personas que no viven en la comunidad y que explican los riesgos con base en sus posiciones en el gobierno (reguladores) y 
orientación política. Así mismo, Renn (2003), en dos estudios de caso demostraba el impacto de los valores culturales en el proceso de amplificación versus atenuación de riesgos en Alemania. Pérez-Floriano (2009) a través de un experimento en el que manipulaba cuatro tipos de información, encontró que las personas que apoyan los valores de igualdad y libertad (universalismo) tendían a amplificar el riesgo mientras que las personas que apoyaban valores de poder y seguridad, tendían a atenuar los riesgos laborales.

La teoría de amplificación de riesgos ha sido sumamente utilizada en estudios cualitativos (véase Pidgeon, Kasperson \& Slovic, 2003), así como en algunos estudios correlacionales a través de cuestionarios (e.g., Frewer, Miles, \& Marsh, 2002; Scherer \& Cho, 2003). En particular, Frewer y sus colegas (2002), encontraron que la teoría de amplificación de riesgos sirve para comenzar el diálogo con el público y explicar el impacto potencial de un evento riesgoso.

\section{ACCIDENTES EN LA INDUSTRIA MINERA}

Aunque sólo ocupe 1\% de la población activa mundial, la minería totaliza aproximadamente $5 \%$ de los accidentes del trabajo mortales $(15,000 \mathrm{al}$ año, o 40 por día). No se dispone de datos fidedignos sobre los accidentes, pero su número es muy elevado, como también lo es el de trabajadores que contraen enfermedades profesionales, que padecen una invalidez permanente, o incluso que pierden la vida como consecuencia directa de su trabajo (Oficina Internacional del Trabajo [OIT] 2003).

A escala mundial, $80 \%$ de las muertes y accidentes en esta industria fueron en China. En dicho país, en el periodo de 2000 a 2005 fallecieron entre 5,800 y 7,000 mineros por año. Mientras que el número de accidentes osciló entre 3,000 y 4,300 por año. Estos números nos indican que los accidentes en minas son en su mayoría fatales, lo que revela el pavor que éstos generan en los mineros, familiares, empresarios del ramo y sociedad.

En 1969 en la misma región mexicana, en las minas de Barroterán, más de 150 mineros del carbón fallecieron cuando una explosión hizo colapsar los túneles en la mina del estado de Coahuila. También en este estado el 29 de septiembre del 2001 estalló la mina La Morita, donde 
perdieron la vida 12 trabajadores y cuatro meses después, el 23 de enero de 2002, se derrumbó La Espuelita, donde fallecieron 13 personas. El último de los accidentes sucedidos en México ocurrió el 19 de febrero de 2006, en la mina de carbón Pasta de Conchos, la que será analizada en este trabajo, cuya explosión dejó 65 muertos.

\section{METODOLOGÍA}

El método utilizado para recabar información fue el de investigación hemerográfica durante el periodo del 19 de febrero al 30 de junio de 2006; se analizaron 240 artículos publicados en los siguientes medios impresos: El Universal, La Jornada y Crónica, y las revistas Proceso y Revista Mexicana de Comunicación. Se analizó el contenido (Casetti \& Di Chio, 1999) de todos los artículos que tuviesen las palabras clave: mina Pasta de Conchos, accidente, minería.

Cabe hacer notar que la teoría de amplificación de riesgos ha tenido el mayor impacto en el análisis del rol de los medios de comunicación en el proceso de amplificación versus atenuación de riesgos (Flynn, Slovic \& Kunreuther, 2001). La información sobre este caso se complementó con dos entrevistas a informantes clave de Minera México con un formato de entrevista no estructurada.

\section{INDUSTRIAL MINERA MÉXICO Y MINA PASTA DE CONCHOS}

La mina Pasta de Conchos es propiedad de Minera México S.A. de C.V. una de las compañías mineras más grandes en México, la cual produce cobre, zinc, plata, oro y molibdeno, operando a través de subsidiarias que se agrupan en tres unidades separadas.

La Unidad Industrial Minera México está conformada por siete minas subterráneas localizadas en la parte central y norte de México; tiene operaciones de proceso industrial del zinc y cobre en San Luis Potosí, San Martín -la mina subterránea más grande de México-, y Charcas, la mina de producción más grande de México.

Además incluye operación de minado de carbón en el noreste de México: Tajo Sur y Pasta de Conchos, esta última ubicada a 10 kilóme- 
tros al oeste de Nueva Rosita, Coahuila y cuya producción en 2005 fue de 257, 016 toneladas (Grupo México, s/f).

\section{PERCEPCIÓN DE RIESGOS}

A partir de 1980, los investigadores se han enfocado a estudiar "el riesgo" desde diferentes perspectivas. El campo de estudio del análisis de riesgos ha crecido rápidamente, enfocándose a la evaluación, administración, propagación, percepción de riesgos (Slovic, 2002). Dentro de las preguntas que responde en el análisis de riesgos encontramos situaciones como las respuestas de la población frente a desastres naturales e industriales (López-Vázquez, 2001; López-Vázquez \& Marván, 2003); riesgos ambientales (e.g., Bronfman, López-Vázquez \& Dorantes, 2009; Bronfman, López-Vázquez, Gutiérrez \& Cifuentes, 2008); seguridad industrial, diferencias nacionales en valores culturales (Pérez-Floriano, 2009; Pérez-Floriano, Flores \& MacLean, 2007; Pérez Floriano \& Gonzalez, 2007).

\section{TEORÍA DE AMPLIFICACIÓN SOCIAL DE RIESGOS}

La amplificación social del riesgo se refiere al fenómeno por el cual los procesos de información, las estructuras institucionales, el comportamiento de los grupos sociales y las respuestas individuales dan forma a la experiencia social del riesgo y sus consecuencias (véase Figura 1). Los sistemas de información y las características de respuesta de las personas son los elementos esenciales en la amplificación que determinan la naturaleza y magnitud del riesgo (Kasperson et al., 1988).

Los sistemas de información amplifican el riesgo de dos maneras: primero, intensificando o debilitando las señales que forman parte de la información que las personas y los grupos sociales reciben del riesgo. Segundo, filtrando las señales haciendo énfasis en las características del riesgo y su importancia.

Las personas e instituciones actúan como "estaciones", procesan la información sobre el accidente, y cada cual en su rol amplificará o minimizará el evento; estas personas o instituciones pueden ser especialistas que comunican el riesgo: institución que maneja el riesgo, medios de 
comunicación, organizaciones activistas, líderes de opinión o de grupos sociales, o agencias públicas. Estas "estaciones” generan y transmiten la información a través de diversos canales de comunicación. También cada receptor de la información intesifica o atenúa el riesgo. Los pasos claves de la amplificación son los siguientes:

- Filtrado de información.

- Decodificación de la señal.

- Procesamiento de la información del riesgo.

- Asociación de valores a la información con el propósito de señalar las implicaciones para la gerencia y política.

- Interacción de los grupos para la interpretación de las señales.

- Formulación de conductas para tolerar el riesgo o tomar acciones en contra del riesgo o de quien maneja el riesgo.

- Incorporación de los individuos a un grupo para aceptar, ignorar, tolerar o cambiar el riesgo.

La amplificación de riesgos también tiene efectos secundarios como pueden ser.

- Percepciones mentales y actitudes como apatía social, odio en contra de organizaciones.

- Impactos en las ventas del negocio y en la actividad económica.

- Presión política y social.

- Cambios en la naturaleza del riesgo.

- Cambios en la educación y manejo para operar y manejar respuestas de emergencia.

- Desorden social.

- Cambios en la regulación y monitoreo de riesgos.

- Incremento de costos.

- Repercusiones en instituciones sociales.

El contacto personal con el riesgo es un factor importante en esta teoría, y éste puede llevar a que el individuo exagere (amplificación) o minimice (atenuar) el riesgo. Además, muchos de los riesgos no son experimentados directamente, entonces es cuando las personas se dan 


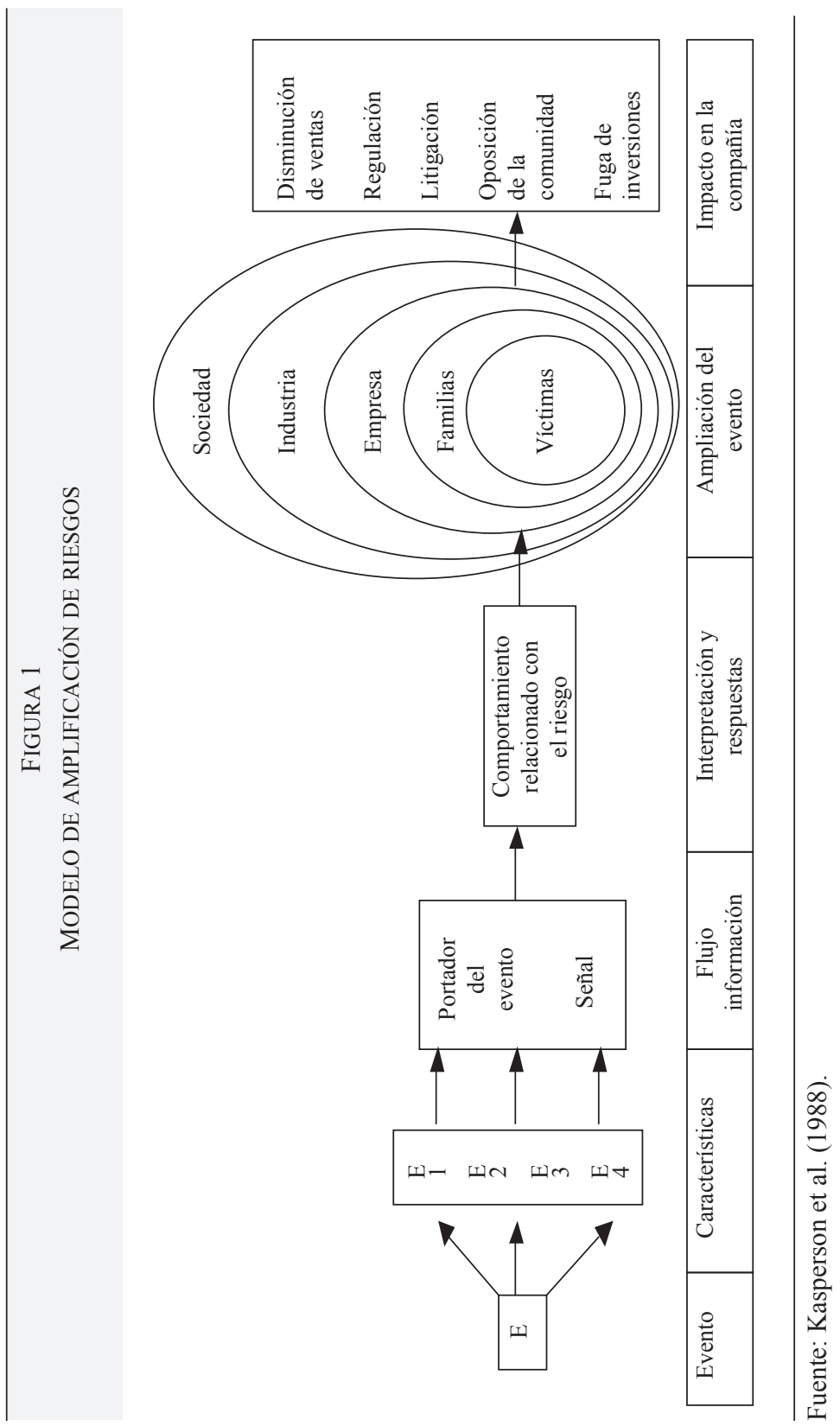


cuenta del riesgo por otras personas o a través de los medios de comunicación. Aquí radica la importancia de los flujos de información en la respuesta social, ya que éstos se convierten en el mayor agente en la intensificación. La información tiene tres atributos que influyen en la amplificación social de riesgos: a) volumen de la información, b) el grado en el que la información es debatida y c) la dramatización, que es indudablemente la forma más poderosa de amplificación.

Es por esto que los canales de información son muy importantes, y sobre todo las relaciones que las empresas - ante una situación de riesgos- deben de tener con los medios y con otras redes de información, sin olvidar las vías de comunicación informales con trabajadores y grupos sociales en general, ya que los rumores que se generan dependen de la percepción y actitud de las personas.

La interpretación y respuesta a la información es la segunda instancia más importante en la amplificación de riesgos, pues estos envuelven contextos sociales, institucionales y culturales en los que la información es interpretada de forma distinta por cada grupo. Entre los principales procesos que explican la activación de la respuesta se encuentran los siguientes:

1. Heurística y valores. La información es interpretada por estos dos procesos mentales, la heurística es la invención de información o simplemente un pensamiento lateral que distorsiona la realidad, y los valores son utilizados para evaluar y dar forma a la respuesta de las personas.

2. Relación con grupos sociales. El riesgo entra en el sector político y social, la naturaleza de estos grupos influye en las respuestas de las personas.

3. Valor de la señal. Las señales acerca del evento de riesgo inician un proceso a través del cual el significado del riesgo es examinado, esto da como resultado los impactos sociales y económicos.

4. Estigmatización. Ideas negativas que se tienen de ciertos grupos. El estigma dentro del evento del riesgo tiene consecuencias significativas sociales y políticas.

Este modelo explica las razones por las cuales pequeños eventos o eventos comunes de riesgo en las organizaciones producen desconcier- 
to social, impactos económicos y diferentes efectos propagados a través del tiempo y espacio (Kasperson et al., 1988).

\section{DESCRIPCIÓN DEL ACCIDENTE}

El derrumbe en la mina se presentó en uno de sus tiros, aproximadamente a 200 metros de la plancha de descenso. ${ }^{3}$ Horas después Grupo México a través de una carta comunicado informó del suceso. Posteriormente, los medios y diversos organismos gubernamentales se dieron a la tarea de informar a la sociedad.

En los primeros días después del derrumbe las acciones de salvamiento fueron continuas e intensivas, en éstas colaboraron elementos especializados, cuadrillas de expertos entrenados y capacitados para el rescate de accidentes en mina. Aún así, las labores transcurrían lentamente ya que después de la explosión, la mina se encontraba altamente explosiva debido a la acumulación de gases, por lo que continuamente el nivel de oxígeno y monóxido de carbono se estaban monitoreando.

De acuerdo con el director general de Minas de la Secretaría de Fomento Económico en Coahuila, Pasta de Conchos era una de las minas más tecnificadas y más seguras. Contaba con un sistema de ventilación en operación continua, lo que daba esperanza a que los 65 mineros estuvieran recibiendo oxígeno. Sin embargo, el gerente de Pasta de Conchos, advirtió que el oxígeno personal con el que cada minero contaba tenía vigencia de 6 horas (Ramos, 2006a).

Conforme pasaban las horas alrededor de 300 familiares, trabajadores y ex trabajadores de la mina permanecieron en los alrededores de la empresa desesperados por la falta de informes. Estas personas externaron a los medios la falta de seguridad de la empresa, sin embargo, el delegado de la Secretaría del Trabajo y Previsión Social (STPS), Pedro Camarillo Adame, señaló que la mina estaba sujeta a supervisión frecuente, y que días antes de la explosión había cumplido con las 43 recomendaciones menores de ajuste de equipo y ademes; por lo que cumplía con las condiciones para operar de forma segura (Proceso, 21/02/2006).

3 Crónica. Documento recuperado el 2 de septiembre de 2006 de http://www. cronica.com.mx/nota.php?id_nota $=277140$ 
Pasados dos días de la explosión, ya eran 200 personas las que participaban en las labores de rescate, y solamente habían logrado avanzar 150 metros, debido a que las condiciones seguían siendo altamente peligrosas para los rescatistas, por lo que el avance se realizaba muy lentamente (50 metros cada 4 horas). Industrial Minera México, S. A. (IMMSA) anunció que dos grupos de especialistas de West Virginia EE.UU. fueron contratados para agilizar las acciones de rescate.

Este mismo día el presidente de la República, Vicente Fox, emitió un comunicado en el que ofreció apoyo material y humano para el rescate, y se dijo que el gobierno federal proveería de la ayuda que fuese necesaria (Suverza \& Fernández, 2006). Para esta fecha ya se encontraban en la mina autoridades que representaban diversos organismos gubernamentales, como la STPS, el Instituto Mexicano del Seguro Social (IMSS), la Secretaría de Salud (SSA) y la Secretaría de Desarrollo Social (SEDESOL), con la finalidad de proporcionar la ayuda necesaria.

Por otra parte, el secretario general del Sindicato Nacional de Trabajadores Mineros, Metalúrgicos y Similares de la República Mexicana (SNTMMSRM), Napoleón Gómez Urrutia, aseguró que se iba a proceder a la investigación de la explosión destacando las siguientes condiciones como causales del accidente: a) se habían reportado fugas de gas, b) el incidente pudo haber sido la consecuencia de negligencia y fallas humanas y c) de los 65 trabajadores atrapados, 25 eran sindicalizados, 4 empleados de confianza y el resto contratistas, mismos que no tenían prestación alguna ni capacitación (Ramos, 2006b).

Los líderes religiosos tuvieron un papel muy relevante para el apoyo de familiares y amigos de los mineros, estando con ellos gran parte del tiempo. También ejercieron su opinión pidiendo apoyo a las autoridades.

Por la noche del 21 de febrero, en conferencia de prensa, el jefe del equipo de rescate de la empresa minera, Josué Beltrán, afirmó que era factible que en horas se pudiera llegar a varios de los mineros atrapados, quienes pudieron haberse refugiado en el cuarto de herramientas, zona segura ya que ahí se respiraba aire fresco y la densidad de gas metano era menor. Los familiares reclamaron mayor veracidad en la información, ya que consideraban que los directivos de la empresa no habían sido realistas en cuanto a la expectativa de encontrar a los trabajadores con vida. 
Después de dos derrumbes, las actividades de rescate fueron suspendidas, lo que provocó en los familiares un enardecimiento tal que intentaron entrar a las instalaciones de la mina, pero desistieron cuando frente al acceso principal se atrincheraron decenas de elementos del ejército y de la Policía Federal Preventiva (PFP). Este incidente se suscitó cuando el titular de la STPS, salía de las instalaciones de la empresa y las familias exigieron un reporte real sobre la situación. Así mismo, el funcionario prometió aplicar las sanciones legales que correspondieran a Industrial Minera México por el accidente (Urrutia \& Ramos, 2006). La tragedia minera atrajo de inmediato la atención de la prensa internacional, por lo que al poblado arribaron reporteros, fotógrafos, camarógrafos y técnicos de empresas de comunicación de distintas partes del mundo.

Pasados cinco días del evento, las esperanzas de encontrar a los mineros vivos se estaban extinguiendo, más derrumbes interrumpían las actividades de recate y se sumaban más rescatistas, pero no se lograba hacer contacto con los mineros atrapados.

Mientras tanto, diversos actores políticos del país pedían a la Secretaría de Economía cancelar concesiones de minas inseguras y una mejor situación laboral para los trabajadores mineros.

El 24 de febrero se recibieron los primeros análisis realizados por técnicos estadounidenses, los cuales determinaron una composición de hasta $40 \%$ de gas en el aire, lo cual mataría cualquier esperanza de vida en el interior; se agregó que se desconocía si las mismas condiciones reinaban en toda la mina. Debido a estas adversidades, en las labores de rescate se avanzaron sólo 3 metros ("Se suspenden por tres días las labores de rescate de mineros", 2006).

Los medios televisivos, luchando por exclusivas sangrientas comenzaron a hablar de muertos, lo cual provocó pánico y un movimiento social agresivo por parte de los familiares.

El mismo día el gobierno federal e IMMSA acordaron suspender las labores de búsqueda durante tres días, al advertir que las elevadas concentraciones de gas metano en la cavidad podrían poner en riesgo la seguridad de los rescatistas. La ira que esto provocó en los familiares tuvo un efecto expansivo entre quienes llevaban seis días aferrados a la esperanza de encontrar vivos a los mineros. 
Varios periodistas escribieron diversas columnas en las que externaban que la explosión de la mina puso de manifiesto la inexistencia de un régimen de derecho en el ámbito laboral, después de que se dio a conocer que les darían a las familias 750,000 pesos de indemnización.

El 25 de febrero, el papa Benedicto XVI envió un mensaje a los familiares de los mineros atrapados en los que mencionó que pidió a Dios por los trabajadores y consuelo y esperanza a ellos. ${ }^{4}$

A siete días de haber transcurrido la explosión, el secretario del trabajo, Francisco Javier Salazar Sáenz; el gobernador del estado de Coahuila, Humberto Moreira, y el presidente de la empresa minera, Francisco Javier García de Quevedo señalaron que la explosión en la mina ocurrida el domingo 19 de febrero, fue mixta y simultánea, debido a las altas concentraciones de gas metano, y que afectó la totalidad de las instalaciones subterráneas en donde se registraron temperaturas superiores a los $600^{\circ} \mathrm{C}$. Así mismo, anunciaron el cierre indefinido de la mina, la futura recuperación de los cadáveres y la reubicación del resto de los obreros.

\section{ANÁLISIS BAJO LA TEORÍA}

DE AMPLIFICACIÓN DE RIESGOS

\section{Percepción de riesgo}

Las percepciones del riesgo juegan un papel muy importante en las decisiones que se deben tomar con base en una situación de riesgo latente. Dentro de la industria minera siempre existe un riesgo constante de accidentes o enfermedades provocadas por los diferentes tipos de gases que se aspiran. Sin embargo, hay trabajadores que perciben el riesgo como mínimo, sienten que nunca les pasará nada; como lo señaló uno de los mineros que trabajaban en la mina: "Yo me levanto todos los días para ir a trabajar y en lo único que pienso es en traer dinero a casa". ${ }^{5}$

4 Crónica. Documento recuperado el 8 de septiembre de 2006 de http://www. cronica.com.mx/nota.php?id_nota=228074

5 Crónica. Documento recuperado el 6 de septiembre de 2006 de http://www. jornada.unam.mx/2006/02/22/0361est.php 
Pero también hay trabajadores para los cuales hay un riesgo latente al laborar dentro de una mina de carbón, y miedo a sufrir algún accidente; la esposa de uno de los mineros atrapados, Amalia Sánchez dijo: "Dos días antes de la explosión mi esposo soñó con la muerte". ${ }^{6}$

Juan Manuel Lares, uno de los mineros que lograron escapar de la mina declaró: "El temor lo trae uno desde niño, así con un temblor en las piernas entras a las minas apenas cumples la mayoría de edad: te arriesgas o te mueres de hambre". ${ }^{7}$

Estos son ejemplos claros de los diferentes niveles de la percepción de riesgos, y estos individuos se clasifican en adversos y tomadores de riesgos.

Para medir la percepción de riesgos se han hecho numerosos estudios, entre los que se encuentra el paradigma psicométrico, en el cual las personas hacen juicios cuantitativos acerca del riesgo de distintos objetos o eventos. Estos juicios están relacionados con los diversos factores del riesgo como: beneficios o perjuicios que el riesgo causa a la sociedad, número de muertes al año (Slovic, 1987). Estudios han mostrado que la percepción del riesgo es cuantificable y predecible. Uno de estos estudios, que a continuación se muestra y en el cual se incluyen los riesgos de accidentes en minas de carbón así como las enfermedades que provocan, se relaciona con dos factores: el primer factor es el miedo que causa el riesgo y se define como la falta de control sobre la situación, que ésta termine en una catástrofe o tenga consecuencias fatales; el segundo factor involucra el conocimiento del riesgo.

Como se puede observar en la Figura 2, los riesgos de accidentes de minas de carbón provocan mucho miedo a las personas; es curioso observar que al público le dan más miedo los accidentes en minas que las enfermedades relacionadas con el trabajo en esta actividad como son el cáncer pulmonar y enfisemas. Enfermedades que aquejan en grado desproporcionado a los mineros y causan miles de fatalidades cada año.

6 Crónica, Documento recuperado el 10 de septiembre de 2006 de http:// www.cronica.com.mx/nota.php?id_nota $=227542$

7 Crónica, Documento recuperado el 6 de septiembre de 2006 de http://www. cronica.com.mx/nota.php?idc $=227530$ 


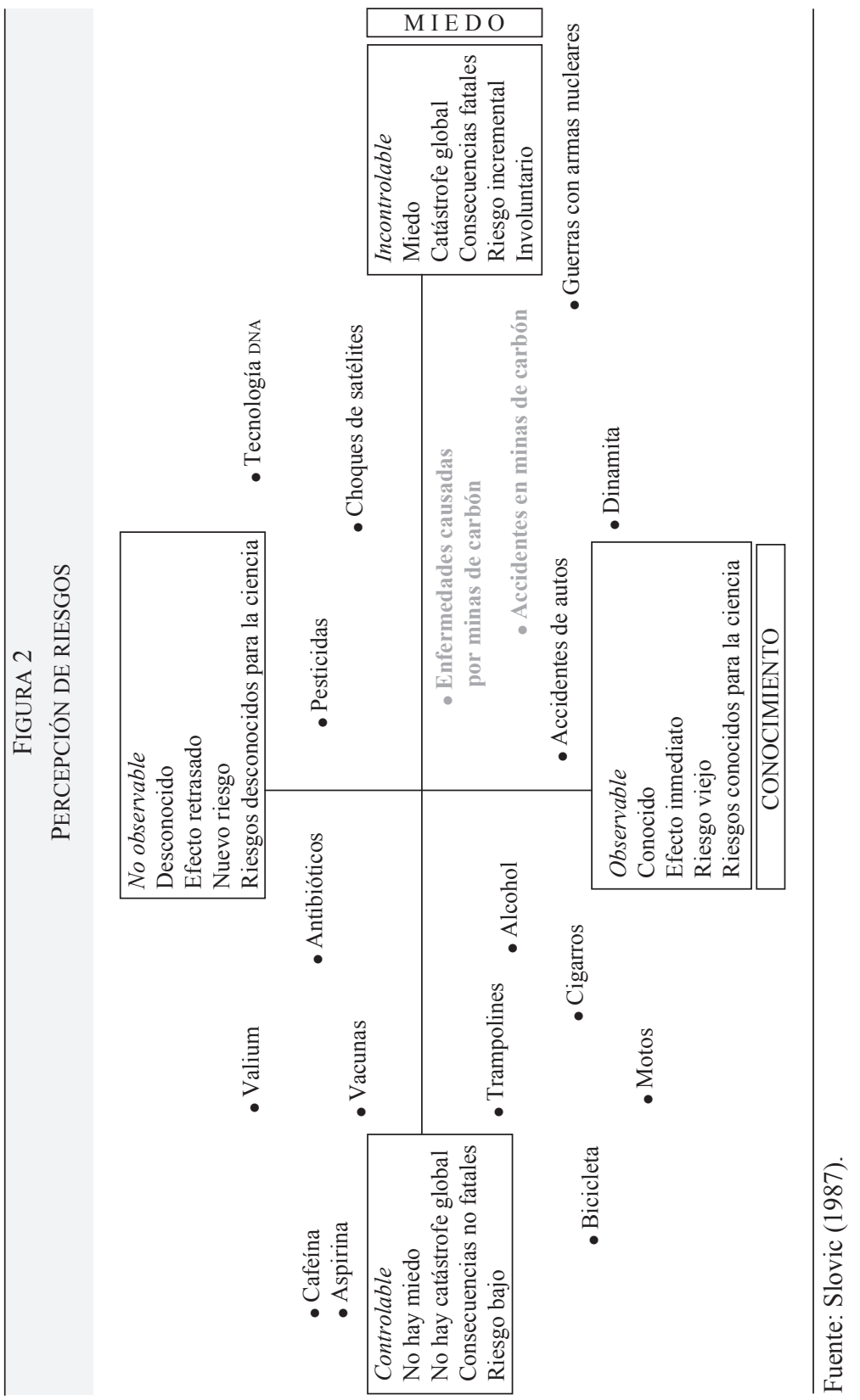


El hecho de que los accidentes en las minas de carbón se consideren catastróficos, con consecuencias fatales, sin posibilidades de reducción, involuntarios e incontrolables es un factor clave en el proceso de amplificación social de riesgos.

\section{AMPLIFICACIÓN SOCIAL DE RIESGOS}

\section{Evento: derrumbe en la mina Pasta de Conchos}

La amplificación social de riesgos es provocada por la ocurrencia de un evento adverso (véase Figura 1). En este caso el derrumbe ocurrido en la mina de carbón Pasta de Conchos, el cual se considera como riesgo conocido, ya que por sus altas probabilidades de consecuencias "catastróficas", causa un alto grado de miedo.

La amplificación social del caso de la mina Pasta de Conchos se dio de la siguiente manera:

1. En la madrugada del 19 de febrero de 2006, a las 2:20 horas, ocurre un derrumbe provocado por una explosión causada por la alta concentración de gas metano. El derrumbe deja un saldo de 65 mineros desaparecidos entre los escombros y 11 heridos.

2. Los primeros comunicados no estuvieron a cargo de la empresa sino que la información se transmitió a través de los familiares de los mineros y de los medios de comunicación.

La muerte de los 65 mineros fue el factor que provocó la propagación de los efectos del derrumbe, ya que el número de muertos hizo que el evento se convirtiera en catastrófico, y con posteriores consecuencias.

Después de diversos estudios en lo referente a las causas de este derrumbe, no se llegó a una conclusión en concreto, se señalan diferentes posibilidades, entre las que se encuentran:

- Falta de control sobre las mediciones de gas metano.

- Mal funcionamiento del sistema de ventilación.

- "Desprendimiento instantáneo", explosión generada antes de que las máquinas registraran los altos niveles de gas metano. 
- "Puenteo de las máquinas", tapar el sensor para que las máquinas excavadoras continúen su trabajo y obtener mayores niveles de carbón.

- Insuficiencia de polvo inerte que contuviera la explosión.

- Falta de soportes laterales para evitar el derrumbe de techo y pared de la mina.

A la muerte de los 65 mineros se suma otro factor importante en la amplificación: el hecho de que los causales del derrumbe no fueron descubiertos, por lo que sus familias y la sociedad en general responsabilizaron a la empresa por el accidente.

La teoría de amplificación de riesgos y su relación con el accidente quedan explicadas en la Tabla 1.

\section{TABLA 1}

\begin{tabular}{ll}
\hline \multicolumn{1}{c}{ Evento } & Derrumbe de la mina Pasta de Conchos \\
\hline Características & - Potencial catastrófico \\
& • Riesgo impuesto por terceros (Empresa \\
& Minera México) \\
& - Actores involucrados no saben cómo con- \\
& trolarlo \\
Flujo de información & Portador del evento: \\
& •Familiares \\
& •Heridos sobrevivientes \\
Interpretación y respuesta & $\begin{array}{l}\text { Furia de los sobrevivientes, familiares, socie- } \\
\text { dad mexicana y comunidad internacional }\end{array}$ \\
Ampliación del impacto & "Estaciones" amplificadoras del evento \\
Impacto en la compañía & Mina cerrada hasta este momento \\
\hline
\end{tabular}

INTERPRETACIÓN DEL EVENTO.

PORTADOR DEL EVENTO Y SEÑALES DE INFORMACIÓN

En una segunda fase de la teoría el evento es transmitido a través de portadores y convertido en señales de información (véanse Figura 1 y Tabla 1). En el caso del accidente Pasta de Conchos, el comportamiento de los medios de comunicación en el suceso, así como los familiares de los mineros, ex trabajadores y trabajadores de la mina, son componen- 
tes esenciales en la influencia en la generación de diversos puntos de vista en la sociedad; sin embargo, los medios de comunicación fueron los encargados de divulgar el suceso, por lo tanto fueron el canal principal a través del cual se dio el flujo de información.

Si consideramos cómo los medios de comunicación muestran el peligro, las personas tienen poca oportunidad de obtener información verídica, ya que los medios tienden a amplificar algunos riesgos y a ignorar otros que son importantes (Friedman, 1994; Kasperson et al., 1988; Renn, 2003; Sandman, 1994; Slovic, 1996). En el caso de la mina Pasta de Conchos los medios no necesitaron ahondar mucho, ellos fueron juez y parte de un evento cuyas implicaciones fueron sin precedente.

Desde el momento en que se hizo público el estallido en el interior de la mina y el recuento de los mineros rescatados, así como de los 65 que permanecían sepultados por miles de toneladas de piedra, carbón, tierra y otros materiales, comenzó la competencia de los medios electrónicos por difundir rápidamente información de primera mano. Las diferentes televisoras del país hicieron de la tragedia una ola mediática susceptible de ser explotada en grandes proporciones.

Los medios impresos no quedaron a la zaga, aunque durante los primeros tres días del lamentable suceso la información procesada tuvo como sustrato reportes de sus corresponsales en aquella entidad federativa norteña y en los despachos de las agencias de noticias nacionales e internacionales.

Del manejo de nota roja propiamente dicho, televisoras y periódicos, así como emisoras radiofónicas, pasaron al plano de la crónica de color y el reportaje: la distribución de alimentos entre los familiares, los servicios religiosos, las esperanzas de las familias por hallar a sus parientes con vida, el equipo sofisticado de los rescatistas, la llegada de los técnicos estadounidenses de apoyo y su impresionante tecnología de punta, la historia de los pueblos mineros de la región fueron los principales temas para los medios de comunicación, dejando de lado la esencia del accidente. 8

$8 \quad$ Pasta de Conchos y los periplos de los medios. Documento recuperado el 9 de septiembre de 2006 de www.mexicanadecomunicacion.com.mx/Tables/ RMC/cuitlahuacarroyo06/pasta.html 


\section{AMPLIFICACIÓN DE RIESGOS Y GRUPO MÉXICO}

Durante los primeros días después de la explosión parecía que los medios de comunicación, y otras autoridades no pertenecientes a la empresa fueron los principales portadores de información. Parecía que las autoridades de la compañía estaban desaparecidas, y salieron demasiado tarde a la luz pública, ya cuando la información había sido interpretada y distorsionada; cuando los medios los habían responsabilizado y condenado completamente de lo ocurrido.

El manejo de la información y de las comunicaciones por parte del sindicato minero IMMSA fue carente. Como lo menciona Juan Rebolledo Gout, vicepresidente de relaciones internacionales de Grupo México: "Lamentablemente no contábamos con un plan de comunicación; realmente no estábamos preparados, nos agarró por sorpresa y en domingo" (Entrevista personal, 26 de noviembre, 2006), lo cual influyó en la distorsión de la información y en la amplificación del evento.

Seis días después de la explosión, comenzaron las contradicciones entre el secretario de la STPS, Francisco Javier Salazar, los técnicos de la empresa Grupo México, concesionarios de la mina y el propio gobernador Moreira; esa parte hizo a los medios retomar la línea policíaca de la información, obviando aspectos sociales medulares, lo que provocaba una sensación de incertidumbre en la sociedad, así como un gran enojo a los familiares que esperaban información acerca de la situación de los mineros enterrados.

Otros portadores de información, aunque en menor magnitud fueron: el gerente de la mina, Rubén Escudero; el gobernador del estado de Coahuila, Humberto Moreira, y el director de Protección Civil Estatal, Sergio Robles. El gerente de la mina no fue el principal informador, ya que se vio opacado por los comentarios que hicieron el director de Protección Civil y el gobernador del estado. A medida que pasaba el tiempo el gerente fue perdiendo el control de la situación y fue pasando a segundo plano en lo que se refiere al flujo de información, lo que provocó que el manejo de la misma saliera de control de la empresa y se quedara en manos de terceros. Nosotras hacemos aquí énfasis en que fue un grave error por parte de Rubén Escudero, gerente de la mina, no aparecer a la luz pública y dejar que otros funcionarios tomaran el 
personaje principal que no les correspondía, y menos ante la necesidad de los familiares y la sociedad en general de obtener información fiable y creíble. La información emitida por los medios como por los diferentes portadores fue en general una tarea de encontrar culpables -de deslindar culpabilidad-más que de informar. Es importante resaltar que las empresas deben siempre tener un plan de relación con los medios para controlar en cierta medida la información, es una tarea difícil pero indispensable para que situaciones en crisis no queden en manos de ajenos que lo único que dan a conocer son interpretaciones de los hechos (Pérez-Floriano, 2004).

La confianza en comunicación de riesgos es de suma importancia en las relaciones de la empresa con sus trabajadores, accionistas y la sociedad (Pérez Floriano, Flores \& MacLean, 2007).

Existieron momentos en que la comunicación pasó a segundo plano para la empresa, por ejemplo las primeras 12 horas después del accidente, en donde nadie salía a informarles a los familiares los nombres y el número de mineros enterrados, así como la situación de los mismos.

De manera más sintetizada se puede decir que la amplificación de riesgos de este evento se debió a que los distintos grupos involucrados en el siniestro actuaron como estaciones de información llevando al fenómeno de la amplificación y después al de la acción colectiva; los grupos fueron los siguientes:

1. Familiares de los mineros. La angustia y desesperación de estos actores fue plasmada por los medios de comunicación; los familiares exigían que sus seres queridos fueran rescatados. Esta exigencia hizo eco en otros actores que la tomaron como suya.

2. Autoridades de la empresa. El gerente de la mina, así como directivos de Grupo México salieron en distintas ocasiones a dar informes a los medios y a los familiares de los mineros fallecidos. Así mismo, crearon una página de Internet especialmente para informar a la sociedad acerca de las actividades de rescate que se estaban llevando a cabo. Como resultado de la falta de un plan de comunicación en crisis esta fuente de información pasó a segundo plano de importancia y perdió el control de la situación. Entre los principales actores de la empresa están: 
- Rubén Escudero, gerente de la mina Pasta de Conchos.

- Francisco Javier García de Quevedo, presidente del Consejo de Administración de IMMSA.

3. Especialistas en accidentes en minas. Técnicos no sólo de México sino de todo el mundo dieron su opinión acerca de las posibles causas del derrumbe. Los actores de este ámbito fueron:

- Sergio Robles, director de Protección Civil del estado de Coahuila, quien pareció el principal vocero de las actividades de rescate de la mina.

- Armando Díaz, coordinador de las operaciones de rescate, apareció públicamente un par de veces solamente para dar informes técnicos del recate.

- Luis Chávez, director general de Minas de la Secretaría de Fomento Económico en Coahuila.

4. Familiares y líderes sindicales. El líder de los mineros se encargó de hacer énfasis en la culpabilidad de la empresa y en la carencia de las condiciones laborales de los mineros. Portador principal:

- Napoleón Gómez Urrutia, secretario general del SNTMMSRM.

5. Lideres religiosos. Exigían a las autoridades solución del problema y daban consuelo a familiares de los mineros. Primero de manera local a través de Alfonso Garza, obispo de Piedras Negras Coahuila; después nacional; mediante el cardenal Norberto Rivera, y por último, internacional, con el mensaje del Papa Benedicto XVI.

6. Líderes políticos. Diferentes autoridades externaron la necesidad de modificar las leyes de seguridad, asimismo, ofrecían apoyo a las familias. Los principales:

- Vicente Fox, presidente de México.

- Francisco Salazar, secretario de la STPS.

- Humberto Moreira, gobernador del estado de Coahuila.

- Napoleón Gómez Urrutia, secretario general del SNTMMSRM.

Entre estos actores políticos se desató una serie de conflictos, por diferentes versiones de las investigaciones del accidente.

Estas "estaciones", de acuerdo con nuestra teoría base, contribuyeron a la amplificación de riesgos. La información que estos generaron y generan, así como el potencial de impacto del accidente está directa- 
mente ligada con las interpretaciones y percepciones de riesgo de las víctimas y sociedad en general.

\section{PROPAGACIÓN DEL IMPACTO}

La propagación del impacto dentro del modelo de Kasperson et al. (1998) se refiere a la manera en que se van esparciendo los sucesos como ondas que genera una piedra al aventarla en el agua; por lo cual se hace una breve descripción de la secuencia de los impactos. En el siguiente apartado se analizarán los impactos como tales.

Las reacciones de la sociedad en lo referente al accidente en la mina Pasta de Conchos es un perfecto ejemplo del paradigma de la amplificación de riesgos. El accidente, siendo no tan catastrófico como los sucedidos en otros países en las minas de carbón, como en China, provocó sentimientos, pensamientos, controversias e incluso propuestas de cambios a las leyes de seguridad en las minas que puedan cambiar el funcionamiento de dicha industria. Ésta es la parte sustancial de la teoría de amplificación de riesgos. El impacto económico del accidente fue tal que a la fecha la mina se encuentra cerrada provocando pérdidas de millones de dólares anuales además de los millonarios gastos de indemnización a los familiares de los mineros fallecidos. Las ondas de propagación del accidente llevaron incluso a una breve huelga nacional de los trabajadores de esta industria. En el nivel social algunas de las repercusiones fueron reformas a la ley.

Inmediatamente ocurrido el derrumbe, el primer impacto que tuvo fue el enterramiento de 65 mineros y 11 heridos. Éstos últimos lograron salir de la mina aproximadamente cuatro horas después de sucedida la explosión y fueron llevados directamente al hospital. Sin embargo, los 65 mineros quedaron atrapados al interior de la mina, a 2 kilómetros de profundidad.

Pareciera cualquier otro accidente laboral, en el cual los impactos simplemente llegan a las víctimas, sin embargo cientos de familiares se vieron directamente afectados por este suceso, tanto personal como económicamente, ya que en la mayoría de los casos el hombre minero era el que mantenía el hogar. Esperando noticias sobre lo sucedido, los familiares permanecieron fuera de la mina aproximadamente una semana, la in- 
formación que les hacían llegar era realmente poca, o incierta, por lo cual los familiares se creaban sus propias ideas acerca de los sucedido dentro de la mina, provocando un ambiente de estrés, que posteriormente desembocó en una furia en contra de la empresa. Tal desorden provocó que el ejército entrara a las instalaciones de la mina para poder controlarlo; todo esto generado por la falta de un plan de comunicación de riesgos que tranquilizase a las personas. El objetivo de la comunicación de riesgos es establecer un diálogo entre dos partes que deseen informarse o discutir sus opiniones sobre un riesgo real o percibido en situaciones en donde la preocupación es alta y la confianza es baja (Pérez-Floriano, 2004).

Por ser domingo fue difícil localizar a autoridades de Grupo México, como lo señaló Juan Rebolledo Gout; los encargados llegaron posteriormente a la mina faltos de información y sin imaginar la magnitud del accidente (Entrevista personal, 26 de noviembre, 2006). Mucho menos esperaban que el suceso fuera a llegar a una magnitud tal que la empresa no sólo enfrentó grandes pérdidas económicas, sino que también tuvo que responder por demandas laborales.

Como si lo anterior fuera poco, este accidente en menos de un mes paralizó la industria minera y metalúrgica del país, causando pérdidas multimillonarias. Este suceso fue un pretexto tomado por el sindicato para desatar un paro en toda la industria; paro que causó no solamente cierre de minas, sino de industrias metalúrgicas en todo el país.

En el nivel social, posterior a la furia y profundo enojo de los familiares de los 65 mineros, no sólo los afectados por el accidente, sino la sociedad en general, así como diversos actores políticos y sociales de México exigieron condiciones de trabajo más seguras en el sector minero, por lo que se llevaron al Congreso diferentes propuestas para hacer reformas a diversas leyes como: ley federal del trabajo, ley del IMSS y ley minera; reformas donde se definían medidas de seguridad y condiciones de trabajo para los mineros, a fin de evitar que se repitan tragedias como la registrada en Pasta de Conchos.

El derrumbe en la mina tuvo diferentes tipos de impactos, entre los que se encuentran:

1. Pérdida en las ventas. La mina Pasta de Conchos dejó de producir 14,000 toneladas de carbón lavado por semana. Las ventas anuales de la mina superaban los 50 millones de dólares y era uno de los ya- 
cimientos más importantes de la región carbonífera. ${ }^{9}$ Hasta agosto de 2010, la mina todavía no reanudaba operaciones.

2. Regulaciones. Tras el derrumbe, tanto diputados locales como federales se dedicaron a crear una serie de propuestas de modificación a las leyes laborales, para poder tener mayor seguridad y control sobre las operaciones en la industria minera. Normas de seguridad que garanticen la integridad de los trabajadores. Las leyes a reformar: ley federal del trabajo, ley del IMSS y ley minera.

3. Conflictos legales. Grupo México se vio involucrado en demandas laborales y en procedimientos para pagar indemnizaciones a los familiares de los mineros fallecidos en las minas; cabe resaltar que esto también implicó un costo económico, del cual no se tienen datos fidedignos.

4. Daños sobre propiedad. La rehabilitación de la mina Pasta de Conchos no pudo ser terminada en un año siguiente al suceso.

5. Huelgas. Grupo México y otras compañías mineras y metalúrgicas sufrieron paros laborales por más de dos meses.

6. Inversionistas. A causa de las huelgas vividas por Grupo México (2006) sus acciones perdieron $4.65 \%$ en dos meses.

7. Cierre de fuentes de trabajo. Se cerraron varias minas en el país, y en consecuencia se perdieron más de 2,000 empleos en la industria minera. 10

8. Desorden social. Familiares de los mineros muertos se apoderaron de las instalaciones administrativas de IMMSA, en exigencia de informes. El ejército mexicano tuvo que tomar las instalaciones para controlar a las 400 personas que exigían información acerca de los fallecidos.

\section{CONCLUSIONES Y RECOMENDACIONES}

Este artículo contribuye al acervo de investigación en México al introducir la teoría de amplificación de riesgos, misma que puede ser útil

9 Mina Pasta de Conchos. Documento recuperado el 29 septiembre de 2006 de http://www.pastadeconchosfacts.com/nuevo/index.htm

10 Crónica. Documento recuperado el 3 mayo de 2006 de http://www.cronica. com.mx/nota.php?id_nota $=23538$ 
para explicar el por qué ciertos eventos con potencial catastrófico llevan a la sociedad a reaccionar, mientras que otros, a pesar de contener un alto costo de vidas humana, sociales y económicas, son prontamente relegadas por los miembros de la sociedad.

La teoría de amplificación de riesgos es útil para explicar y predecir la reacción del público a un riesgo con potencial de crisis y puede complementar modelos de manejo de crisis como el propuesto por Augustine (2000) en el que se analizan los peligros que pueden incidir en el manejo de la organización y se elaboran escenarios de manejo de crisis.

El análisis del accidente sucedido en la mina Pasta de Conchos bajo la teoría de amplificación de riesgos nos ha demostrado que los impactos que puede tener un evento de riesgo en una empresa pueden llevarse a magnitudes impredecibles si no se cuenta con un plan de comunicación de riesgos y manejo de crisis.

La conceptualización de estos impactos bajo el modelo de Kasperson et al. (1988), ayudaría a las empresas a construir modelos de decisiones para valorar la adaptación de medidas preventivas de riesgos, y no soluciones de grandes problemas.

Este es un claro ejemplo de que las empresas no solamente deben de crear planes de crecimiento, sino también planes de comunicación en crisis, sobre todo en industrias en que las crisis y accidentes son muy frecuentes y por lo tanto predecibles. Esto sin eludir la principal obligación de las empresas donde debe predominar un clima de seguridad laboral, enfocado en la preservación de la vida de sus trabajadores en un marco de sustentabilidad.

La mina Pasta de Conchos perdió recursos financieros, su reputación, y la de sus directivos se afectó por la carencia de control de la situación. Las empresas de alto riesgo o susceptibles de accidentes deben de "administrar el riesgo". La administración del riesgo debe incluir un análisis de los siguientes factores (Slovic, 1987): toma de decisiones, comunicación, seguridad, riesgo aceptable, atenuación. IMMSA no contaba con un plan de comunicación en crisis, lo cual constituyó un factor que provocó que el derrumbe tuviera gran impacto, tanto en la empresa misma como en la política, en el ámbito laboral y en la industria en general.

En lo que se refiere a la toma de decisiones, en una industria como la minería, las empresas deben dedicar parte de sus recursos, tanto mone- 
tarios como humanos, a la planeación de crisis para saber la manera en que se tomarán las decisiones llegado el momento. Esto es una actividad vital que necesita recursos financieros, humanos y un compromiso por parte de la organización. Se sugiere por tanto:

- La seguridad, el tener un sistema de prevención de riesgos y acidentes, así como contar con todas las medidas de seguridad necesarias según la organización lo requiera para evitar este tipo de situaciones que lleven a impactos catastróficos. Seguir todas las regulaciones que la ley exige para evitar futuros problemas legales y dudas por parte de las autoridades y de la sociedad.

- Hacer que este tipo de sucesos se vuelva un riesgo aceptable es una tarea muy difícil, pero recomendable. Las minas deben de hacer conscientes a sus trabajadores y a los familiares que el trabajar dentro de una mina implica riesgos, que a pesar de que se tomen todas las medidas de seguridad se incurre en ellos. Éste es un factor importante que podría hacer que la sociedad aceptara este tipo de accidentes como un riesgo latente e implícito al trabajar en minas, y no como un homicidio, previniendo así la amplificación y la afectación hacia la sociedad y hacia toda la industria.

- La atenuación va ligada a hacer el riesgo aceptable y la asimilación depende de que la organización demuestre con hechos y dichos que constantemente se hacen esfuerzos por remover todos los peligros que pueden afectar la salud de las y los trabajadores, a través de prácticas adecuadas de seguridad e higiene. Esto lleva a tener una reputación sólida dentro de la empresa, misma que eventualmente permea a la comunidad.

\section{Bibliografía}

Augustine, R. N. (2000, diciembre). How to keep a crisis from happening. The underappreciated value of "precrisis management". Harvard Management Update, 6, 1-3.

Bronfman, N., López-Vázquez, E., Gutiérrez V. \& Cifuentes, L. A. (2008). Trust, acceptance and knowledge of technological and environmental hazards in Chile. Journal of Risk Research, 11, 755-773.

Bronfman, N., López-Vázquez, E., Dorantes, G. (2009). An empirical 
study for the direct and indirect links between trust in regulatory institutions and acceptability of hazards. Safety Science, 47, 686692.

Casseti, F. \& Di Chio (1999). Análisis de la televisión. Instrumentos y prácticas de investigación. Colecciones Instrumentos, dirigida por H. Eco. Barcelona: Paidós.

Fernández, H. (2006, 4 de marzo). Pierden por cierre de mina más de $1 \mathrm{mdd}$ a la semana. El Universal. Recuperado el 9 de septiembre de 2006 de http://www.eluniversal.com.mx/estados/60383.html

Flynn, J., Slovic, P., \& Kunreuther, H. (2001). Risk, media and stigma: Understanding public challenges to modern science and technology. Londres: Earthscan.

Frewer L. J., Miles, S. \& Marsh, R. (2002). The media and genetically modified foods: evidence in support of social amplification of risk. Risk Analysis, 22, 701-711.

Friedman, S. M. (1994). The media, risk assessment and numbers: They don't add up. Risk, 5. Recuperado de http://www.fplc.edu/RISK/ vol5/friedman.htm]

Grupo México (2006). Informe anual. México, D. F.: Autor.

Grupo México (s/f). Documento recuperado el 4 de septiembre de 2006 de http://www.gmexico.com.mx/inversionistas/informacion-financiera.php

Kasperson R. E., Renn O., Slovic, P., Brown. H. S., Emel, J., Globe, R., et al. (1988). The social amplification of risk: A conceptual framework. Risk Analysis, 8, 177-187.

López-Vázquez, E. (2001). Risk perception interactions in stress and coping facing extreme risks. Environmental Management and Health, 12, 122-133.

López-Vázquez, E., \& Marván M. L. (2003). Risk perception, stress and coping strategies in two catastrophe risk situations. Social Behavior and Personality, 31, 61-70.

Masuda, J. R. \& Garvin, T. (2006). Place, culture, and the social amplification of risk. Risk Analysis, 26, 437-454.

Oficina Internacional del Trabajo-OIT (2003). La seguridad y la salud en las industrias de los metales no ferrosos: repertorio de recomendaciones prácticas de la OIT. Oficina Internacional del Trabajo. Ginebra. 
Pérez-Floriano, L. (2004). Comunicación de riesgos y seguridad industrial. Dirección Estratégica, 10 (3). 10-13.

Pérez-Floriano, L. (2009). Real and perceived risk among workers from the NAFTA region: A psychological and cultural explanation of safety compliance. Alemania: VDM Verlag.

Pérez-Floriano, L., Flores Mora, J. \& MacLean, J. (2007). Trust in risk communication in organizations in five countries of North and South America. Risk Assessment and Management, 7, 205-223.

Pérez-Floriano L. \& González, J.A. (2007). Risk, safety and culture in Brazil and Argentina: The case of Transinc Corporation. International Journal of Manpower. 28, 403-417.

Pidgeon, N., Kasperson, R. E. \& Slovic, P. (2003). The social amplification of risk. EE.UU.: Cambridge University Press.

Proceso (2006, 21 de febrero). Se agotan expectativas de encontrar vivos a los 65 mineros. Recuperado el 4 de septiembre de 2006 de http://www.proceso.com.mx/rv/modHome/detalleExclusiva/37794

Ramos, L. (2006a, 20 de febrero). Explosión y derrumbe en una mina de Coahuila; 65 obreros atrapados. La Jornada. Recuperado el 2 de septiembre de 2006 de http://www.jornada.unam.mx/2006/02/20/ index.php?section $=$ estados $\&$ article $=038 \mathrm{n} 1$ est

Ramos, L. (2006b, 21 de febrero). Sin avance, el rescate de mineros en Coahuila; zozobra de familiares. La Jornada. Recuperado el 4 de septiembre de 2006 de http://www.jornada.unam.mx/2006/02/21/ index.php? section $=$ estados $\&$ article $=036 \mathrm{n} 1$ est

Renn, O. (2003). Social amplification of risk in participation: Two case studies. En N. Pidgeon, R. E. Kasperson \& P. Slovic (Eds.), The social amplification of risk (pp. 374-401). EE.UU.: Cambridge University Press.

Rosa, E. A. (1998). Metatheoretical foundations for post-normal risk. Journal of Risk Research, 1, 15-44.

Sandman, P. M. (1994). Mass media and environmental risk: Seven principles. Risk, 5.

Scherer, C.W. \& Cho, H. (2003). A social network contagion theory of risk perception. Risk Analysis, 23, 261-267.

Se suspenden por tres días las labores de rescate a mineros. (2006, 24 de febrero). Proceso.com. Recuperado el 3 de febrero de 2010 de 
http://www.proceso.com.mx/rv/modehome/detalleExclusiva/37977 Short, J. F. Jr. (1987). Social dimensions of risk: The need for a sociological paradigm and policy research. American Sociologist, 12, 167-172.

Slovic, P. (1987). Perception of risk. Science, 236, 280-285.

Slovic, P. (1996). Experts must respect and include citizens in decisions on risk. Environmental Health Letter, 1, 7.

Slovic, P. (2002). Perception of risk posed by extreme events. (pp. 1-21). EE.UU.: Decision Research/University of Oregon.

Suverza, A. \& Fernández, H. (2006, 21 de febrero). Lento avance en rescate de mineros; esperan milagro. El Universal. Recuperado el 4 de septiembre 2006 de http://www.eluniversal.com.mx/notas/331894.html

Urrutia, A. \& Ramos, L. (2006, 22 de febrero). Autoridades cavan fosas mientras dan esperanzas a familias de mineros. La Jornada. Recuperado el 6 de septiembre de 2006 de http://www.jornada.unam. $\mathrm{mx} / 2006 / 02 / 22 /$ index.php? section $=$ estados $\&$ article $=036 \mathrm{n} 1$ est

Wilkinson, I. (2001). Social theories of risk perception: At once indispensable and insufficient. Current Sociology, 49 (1), 1-22.

Wynne, B. (1992). Risk and social learning: Reification to engagement. En S. Krimsky \& D. Golding (Eds.), Social theories of risk, 12. Connecticut, EE.UU.: Praeger. 